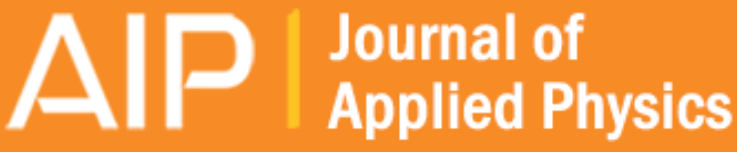

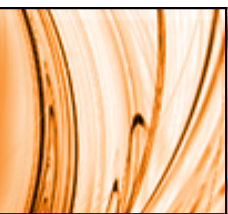

\section{Progressive structural and electronic properties of nano-structured carbon atomic}

chains

D. Usanmaz and G. P. Srivastava

Citation: Journal of Applied Physics 113, 193704 (2013); doi: 10.1063/1.4805047

View online: http://dx.doi.org/10.1063/1.4805047

View Table of Contents: http://scitation.aip.org/content/aip/journal/jap/113/19?ver=pdfcov

Published by the AIP Publishing

\section{Articles you may be interested in}

Bond length and electric current oscillation of long linear carbon chains: Density functional theory, MpB model, and quantum spin transport studies

J. Chem. Phys. 140, 134703 (2014); 10.1063/1.4869858

The first-principles study of oscillating rectifying performance in nanoribbon-chain-carbon nanotube junctions Appl. Phys. Lett. 102, 203106 (2013); 10.1063/1.4807284

Electronic structure and transport properties of sulfur-passivated graphene nanoribbons

J. Appl. Phys. 112, 113710 (2012); 10.1063/1.4768524

Structural, electronic, optical, and magneto-optical properties of Bi12MO20 (M=Ti, Ge, Si) sillenite crystals from first principles calculations

J. Appl. Phys. 110, 083705 (2011); 10.1063/1.3652751

Progressive changes in surface structure and electronic properties on $\mathrm{Si}(001)$ surface by $\mathrm{CaF} 2$ adsorption

J. Appl. Phys. 109, 123708 (2011); 10.1063/1.3597827

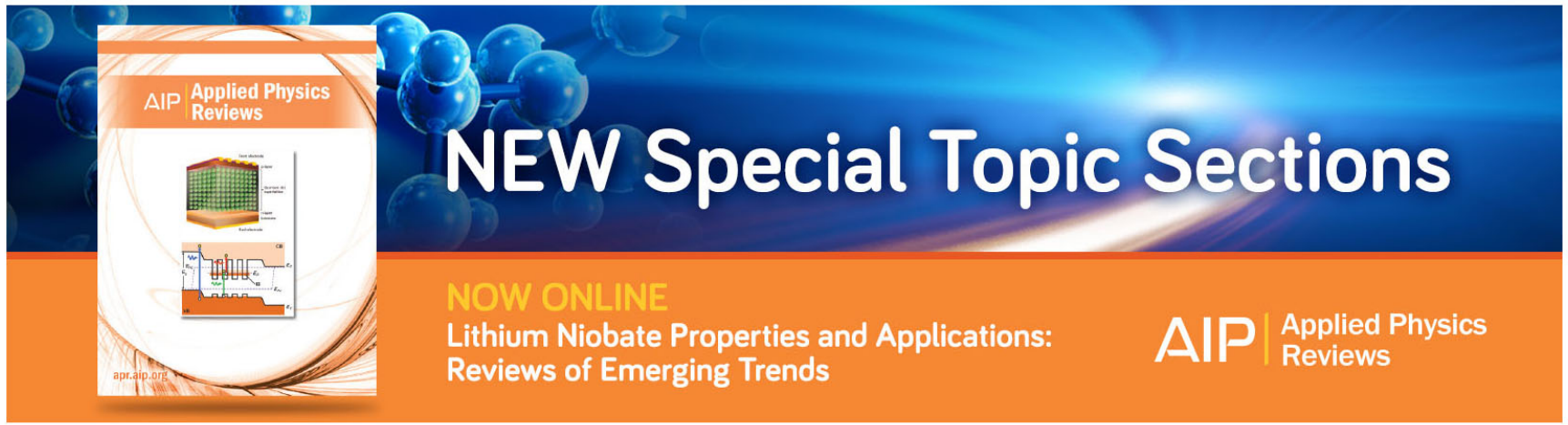




\title{
Progressive structural and electronic properties of nano-structured carbon atomic chains
}

\author{
D. Usanmaz ${ }^{1}$ and G. P. Srivastava ${ }^{2}$ \\ ${ }^{1}$ Physics Program, Middle East Technical University, Northern Cyprus Campus, Kalkanl i, Güzelyurt, KKTC, \\ Mersin 10, Turkey \\ ${ }^{2}$ School of Physics, University of Exeter, Stocker Road, Exeter EX4 4QL, United Kingdom
}

(Received 27 February 2013; accepted 29 April 2013; published online 16 May 2013)

\begin{abstract}
$A b$ initio calculations, based on the planewave pseudopotential method and the density functional theory, have been reported on the changes in the electronic and structural properties of short carbon atomic chains held rigidly between hydrogenated thin armchair graphene nanoribbons ( $N_{a}$-AGNR) of dimer line numbers $N_{a}=4$ and 5 . We have considered chains of several lengths $(n=4-9$ atoms) and with different forms of attachment with the AGNRs. It is found that odd-numbered chains are metallic in nature, with chemical bonding more like $\cdots \mathrm{C}=\mathrm{C}=\mathrm{C}=\mathrm{C} \cdots$ (as in cumulene). Even numbered chains show semiconductor structure when held between 4-AGNR and semi-metallic nature when held between 5-AGNR, in both cases characterized by chemical bonding more like $\cdots \mathrm{C}-\mathrm{C} \equiv \mathrm{C}-\mathrm{C} \equiv \mathrm{C} \cdots$ (as in polyyne). (C) 2013 AIP Publishing LLC. [http://dx.doi.org/10.1063/1.4805047]
\end{abstract}

\section{INTRODUCTION}

Recently, graphite related materials have become the focus of research interest in condensed matter physics. In particular, thin strips of graphene, called graphene nanoribbons (GNRs), exhibit different electronic structure depending on the their width and shape of edges, ${ }^{1-3}$ such as armchair and zigzag. Due to their versatile properties, they are more attractive for device applications such as gas sensors ${ }^{4}$ and spin valves. ${ }^{5}$ In addition, it is much easier to produce and manipulate the GNRs and offer the opportunity to investigate the electronic properties of the carbon nanotubes (CNTs). It is well known that GNRs with armchair edges (AGNR) have similar electronic properties with zigzag CNTs and vice versa. GNRs with armchair shaped edges can be either metallic or semiconducting depending on their widths, and zigzag GNRs (ZGNRs) are metallic with edge states without depending on its widths. ${ }^{1,2,6-10}$ Onedimensional (1D) AGNRs are described by the number of dimer lines, $N_{a}$, across the ribbon width. Calculations based on the density functional theory (DFT) showed that when the width of the ribbon is $N_{a}=3 M-1$ ( $M$ is an integer) AGNRs are metallic. ${ }^{2,11}$ Zero-dimensional AGNRs have length dependence for certain widths, which varies from the 1D-AGNR results. ${ }^{12}$ However, hydrogen terminated 1DZGNRs show flat edge states, which induce a sharp peak of density of states (DOS) at the Fermi level. ${ }^{13}$ Very recently, Lu et $a l .{ }^{11}$ have studied the electronic structures of the Fterminated ZGNRs and AGNRs. According to their findings, because of the $\sigma-\pi$ mixing effect, the non-bonding $2 p$ electrons of F-atoms produce orbital mixing with the $\sigma$ valence electrons, and also F-terminated AGNRs have lower band gaps than H-terminated AGNRs. ${ }^{11}$

Recently, GNRs joined by short carbon chain systems have attracted a great deal of attention after their successful fabrication. These systems can be expected to reveal interesting electronic properties. Controlled energetic electron irradiation technique inside a transmission electron microscope (TEM) has been employed to continuously thin a GNR to produce stable and rigid carbon atomic chains (CACs) between two GNRs. ${ }^{14,15}$ Using this novel experimental technique, Jin et al. ${ }^{14}$ managed to synthesize carbon chains of length up to sixteen atoms and of width down to one atom. Using a first-principles theoretical method, this group further showed that this form of carbon material, i.e., nanostructured carbon wires constrained by two GNRs with zigzag edges, is very stable. In the same year, Chuvilin et al. ${ }^{15}$ successfully transformed GNRs to single CACs under the high resolution transmission electron microscopy (HRTEM) conditions. However, they observed pentagon and heptagon shapes at the GNR edges. Following these experimental achievements, transport properties of GNR-CAC-GNR systems have been receiving great attention. In this respect, the work by Lang and Avouris is noteworthy. ${ }^{16-18}$ Using a similar technique, Shen et al. ${ }^{19}$ have investigated transport properties of ZGNR-ended CAC systems. Because of the metallic properties of ZGNRs, they prefer to use these as leads. Their results suggest that conductance is independent of the structure of the CAC. Also, hydrogen impurities do not change the conductance, but oxygen impurities do reduce it under a low bias. The findings of Lang and Avouris ${ }^{16}$ show that odd-numbered chains have lower resistance (higher conductance) than even-numbered chains due to high DOS at their Fermi level. Xu et al. ${ }^{20}$ have considered different bridge positions for the CAC between ZGNRs and their results indicate that the transport behavior is sensitive to the bridge position and the spin configuration of the leads.

A rigid monatomic carbon linear chain is expected to exhibit two types of bonding: either $\cdots \mathrm{C}-\mathrm{C} \equiv \mathrm{C}-\mathrm{C} \equiv \mathrm{C} \cdots$ (as in polyyne) or $\cdots \mathrm{C}=\mathrm{C}=\mathrm{C}=\mathrm{C}=\mathrm{C} \cdots$ (as in cumulene), as discussed by Jin et al. ${ }^{14}$ An obvious and interesting question is: what kind of bonding exists in a carbon chain constrained by two AGNRs. Calzolari et al. ${ }^{21}$ proposed that the electrical properties of ideal 1D monatomic C chains are 
directly linked to their $\pi$ states. For cumulene type CACs, $\pi$ orbitals are centered on the atoms and lead to metallic system, while the $\pi$ states of polyyne being localized on the triple $\mathrm{C} \equiv \mathrm{C}$ bonds induce a semiconducting behavior. It is, therefore, very important to determine details of the electronic structures and investigate the effect of the number of atoms (in the $\mathrm{CAC}$ ) in the electronic structure.

The present study aims at clarifying the atomic and electronic structures of short carbon atomic chains held rigidly between hydrogenated armchair graphene nanoribbons. We consider chains of several lengths (containing $n=4-9$ atoms) and ending forms $\left(\mathrm{sp}^{2}\right.$ and $\left.\mathrm{sp}^{3}\right)$. The results are analysed in terms of even- and odd-numbered carbon atoms in the chains for AGNR-ended chains to examine the variation of the DOS and band structures around the Fermi level $\left(E_{F}\right)$.

\section{COMPUTATIONAL METHOD}

We carried out $a b$ initio pseudopotential calculations by employing artificially invoked periodic boundary conditions along the width direction of the modelled structures for the CACs as shown in Fig. 1. The electron-electron interaction was considered within the local density approximation (LDA) of the DFT, using the correlation scheme of Ceperley and Alder $^{22}$ as parametrised by Perdew and Zunger. ${ }^{23}$ The electron-ion interaction was considered in the form of normconserving ${ }^{24}$ fully separable pseudo-potentials. ${ }^{25}$ The singleparticle Kohn-Sham ${ }^{26}$ wave functions were expanded in a

plane-wave basis set with the kinetic-energy cutoff 30 Ry. This cutoff was found to be adequate, as calculations with the kinetic energy cutoff 40 Ry did not significantly change the atomic relaxation or the electronic band structure results. Therefore, we performed all calculations with $30 \mathrm{Ry}$. Selfconsistent solutions of the Kohn-Sham equations were obtained by employing six $\mathbf{k}$ points ${ }^{27}$ in the irreducible part of the Brillouin zone for the structures considered. The eigenvalues at the special $\mathbf{k}$ points were thermally smeared by using the Fermi-Dirac distribution function with a broadening factor of $0.05 \mathrm{eV}$.

In our calculations, the CACs were terminated by GNRs with armchair edges and the edge atoms of the latter were passivated by hydrogen. The unit cell included $8 \AA$ vacuum in the $\mathrm{x}$ and $\mathrm{z}$ directions and the AGNRs at the two ends of a CAC were of 4-AGNRs and 5-AGNR. In this article, we mostly investigated the atomic and electronic properties of carbon wires connected between AGNRs with $\mathrm{sp}^{2}$ bonding. However, some $\mathrm{sp}^{3}$ bonding connections are also investigated. Only the atoms in the middle of one of the AGNRs were frozen and all other carbon atoms and the passivating hydrogen atoms were relaxed till forces on them became negligibly small.

\section{RESULTS AND DISCUSSION}

We have performed a series of total energy calculations for short CACs with the number of carbon atoms $n$ ranging

(b)

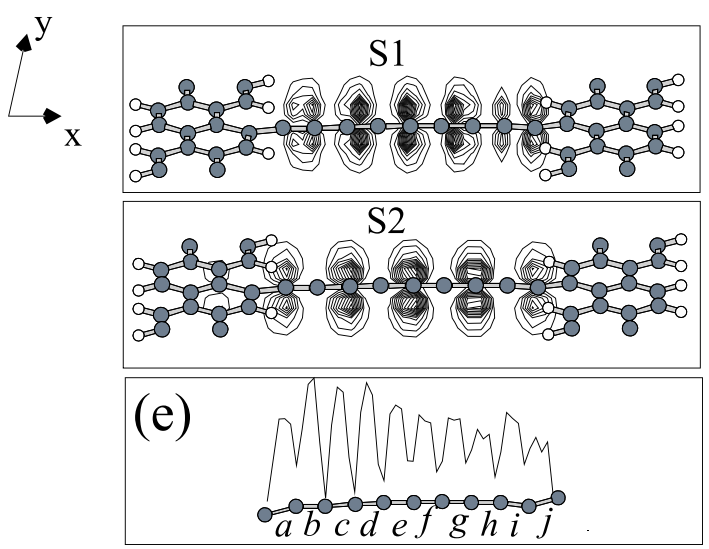

(c)

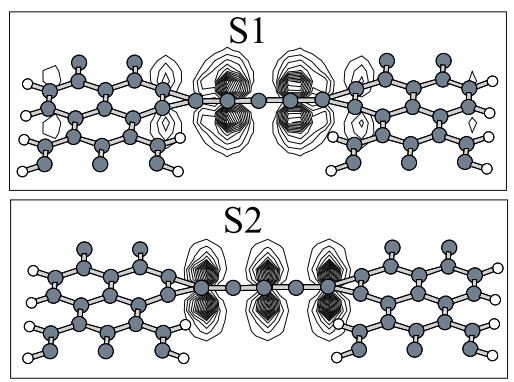

(h)

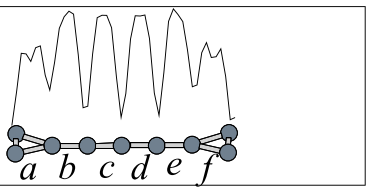

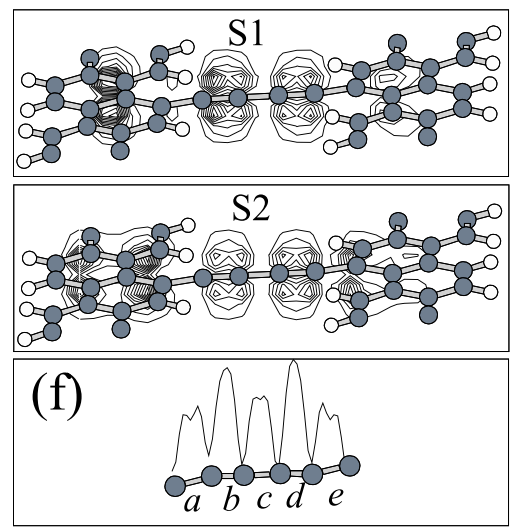

(d)

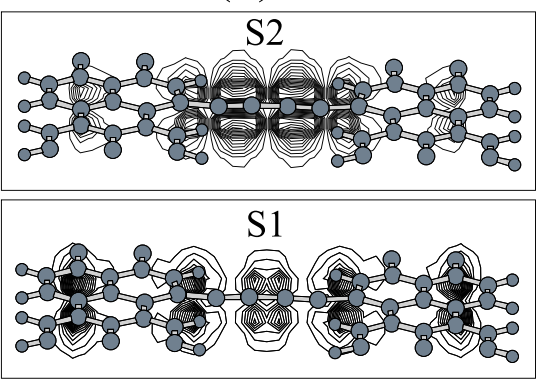

(g)

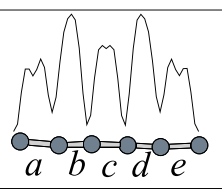

FIG. 1. Relaxed representative geometries for $n$-atom short carbon chains constrained by two AGNRs. Panel (a): a 9-atom chain held between 4-AGNR [referred to as 4AGNR-C $\mathrm{C}_{9}$ in the text); panel (b): an 4-atom chain held between 4-AGNR [referred to as 4-AGNR-C 4 in the text); panel (c): a 5-atom chain held straight between 5-AGNR [referred to as 5-AGNR-C ${ }_{5}-s p^{3}$ in the text); panel (d): a 4-atom chain straight between 5AGNR [referred to as 5-AGNR-C 4 in the text). The upper and lower parts of each panel show charge density plots for $S_{1}$ halfoccupied and $\mathrm{S}_{2}$ unoccupied electronic band states, respectively. The total electronic charge density distribution along the carbon chains is shown as: (e) 4-AGNR-C 9 , (g) 4AGNR-C 4 , (h) 5-AGNR-C $5-s p^{3}$, and (f) 5AGNR-C 4 . 
from 4 to 9 . The chains were held straight between two armchair GNRs (referred to as $\mathrm{N}_{a}-\mathrm{AGNR}-\mathrm{C}_{n}$ ).

Previous DFT calculations showed that AGNRs of width expressed with $N_{a}=3 M-1$, where $M$ is an integer, are metallic. ${ }^{1,7,28}$ However, band calculations showed that the $\mathrm{H}$ - and F-terminated 1D-AGNRs with $N_{a}=5$ and 8 are semiconducting with tiny band gaps, ${ }^{2,11}$ whereas for $N_{a}=3,4,6$, $7 \ldots$, the energy band gap becomes bigger. In this paper, first, we have recalculated the electronic band structure of the $\mathrm{H}$ terminated 1D-AGNRs with $N_{a}=4$ and 5. As can be seen clearly from Figs. 2(a) and 3(a), the electronic band structure of the H-terminated AGNRs shows results similar to those obtained previously for F-terminated ${ }^{11}$ and H-terminated ${ }^{11}$ AGNRs. Our H-terminated AGNRs have higher band gap than F-terminated AGNRs. ${ }^{11}$ It is clear that the electronic band structures (Figs. 2(a) and 3(a)) indicate semiconductor character for 4-AGNR and 5-AGNR, in agreement with the calculation by Lu et al. ${ }^{11}$ Also, 4-AGNR has a bigger band gap of $\mathrm{E}_{g}=2.62 \mathrm{eV}$. To get a clearer understanding, we have calculated the DOS around the Fermi level. Although 4AGNR obviously has a band gap, 5-AGNR exhibits semimetal character with a finite DOS at $\mathrm{E}_{F}$.

The atomic geometry and electronic structure of CACs were studied with these held rigidly between semiconducting 4-AGNRs and also between semi-metallic 5-AGNRs. Table I presents the key relaxed atomic geometry parameters for the odd-numbered and even-numbered CACs. For the 4-AGNR$\mathrm{C}_{n}$ structure, the shortest bond length in odd-numbered chains $(1.28 \AA)$ is shorter than that in even-numbered chains (1.31 $\AA$ ). The longest $\mathrm{C}-\mathrm{C}$ bond length occurs at the ends of a chain (e.g., bond $a,{ }^{29}$ joining the chain to the AGNR), in agreement with the calculations by Jin et al. ${ }^{14}$ The longest $\mathrm{C}$ (chain)-C(AGNR) bond length is $1.50 \AA$ for odd-numbered chain with $n=5$ and 7 and $1.54 \AA$ for even-numbered chain with $n=6$. For both odd-numbered and even-numbered chains, the shortest bond length is established next to the (a)

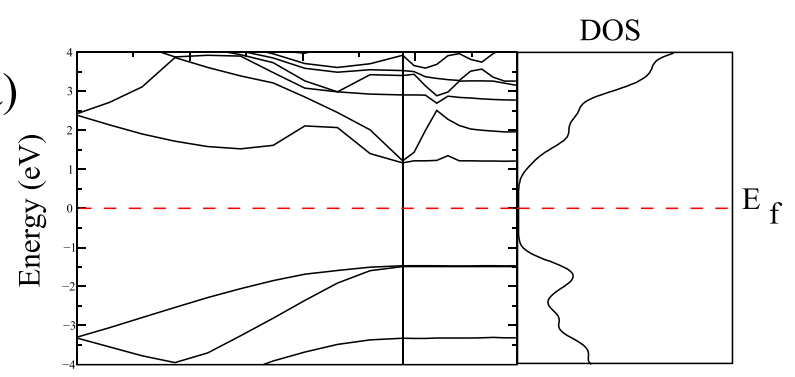

(b)

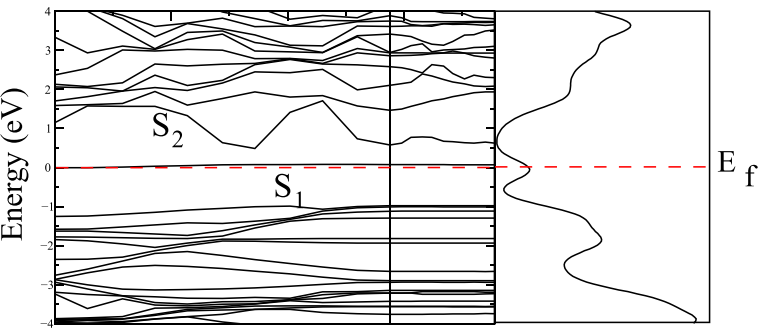

(c)

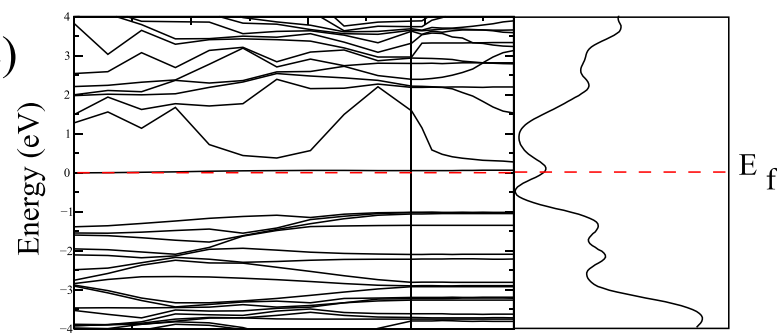

(d)

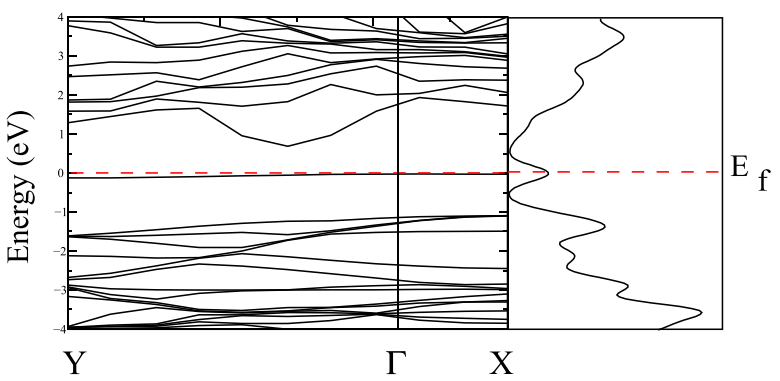

(e)

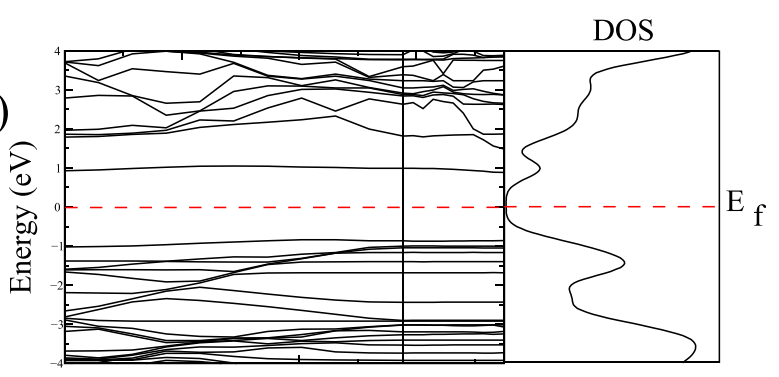

(f)

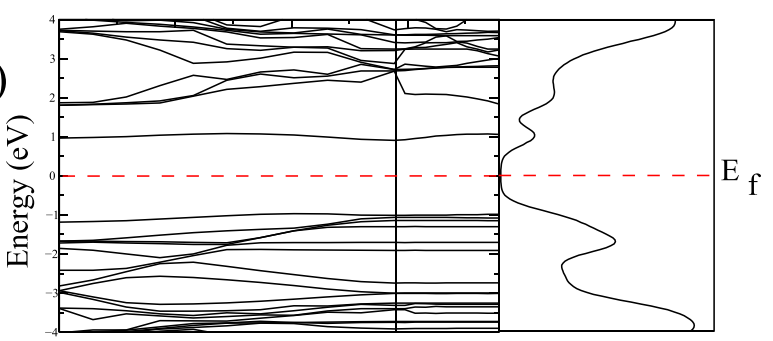

(g)

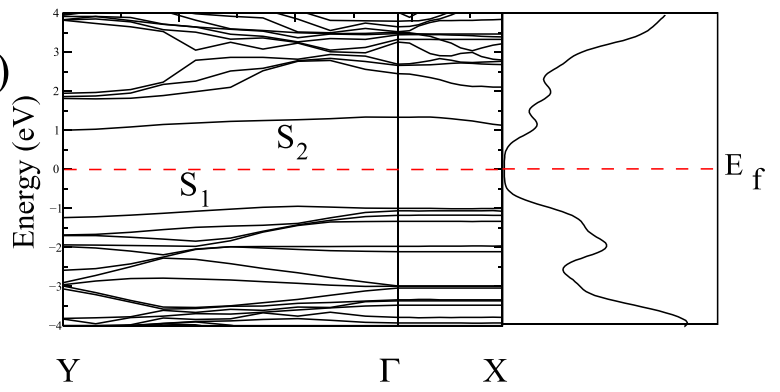

FIG. 2. Density of states around the Fermi level $\left(E_{F}\right)$ for 4-AGNR-C ${ }_{n}(n=4-9)$ with carbon chain of different lengths. For comparison, band structure and density of states of a 4-AGNR are also shown. The Fermi level is set at $0 \mathrm{eV}$. (a) 4-AGNR, (b) 4-AGNR-C 9 , (c) 4-AGNR-C 7 , (d) 4-AGNR-C 5 , (e) 4-AGNR-C 8 , (f) 4-AGNR-C 6 , and (g) 4-AGNR-C . $^{2}$ 

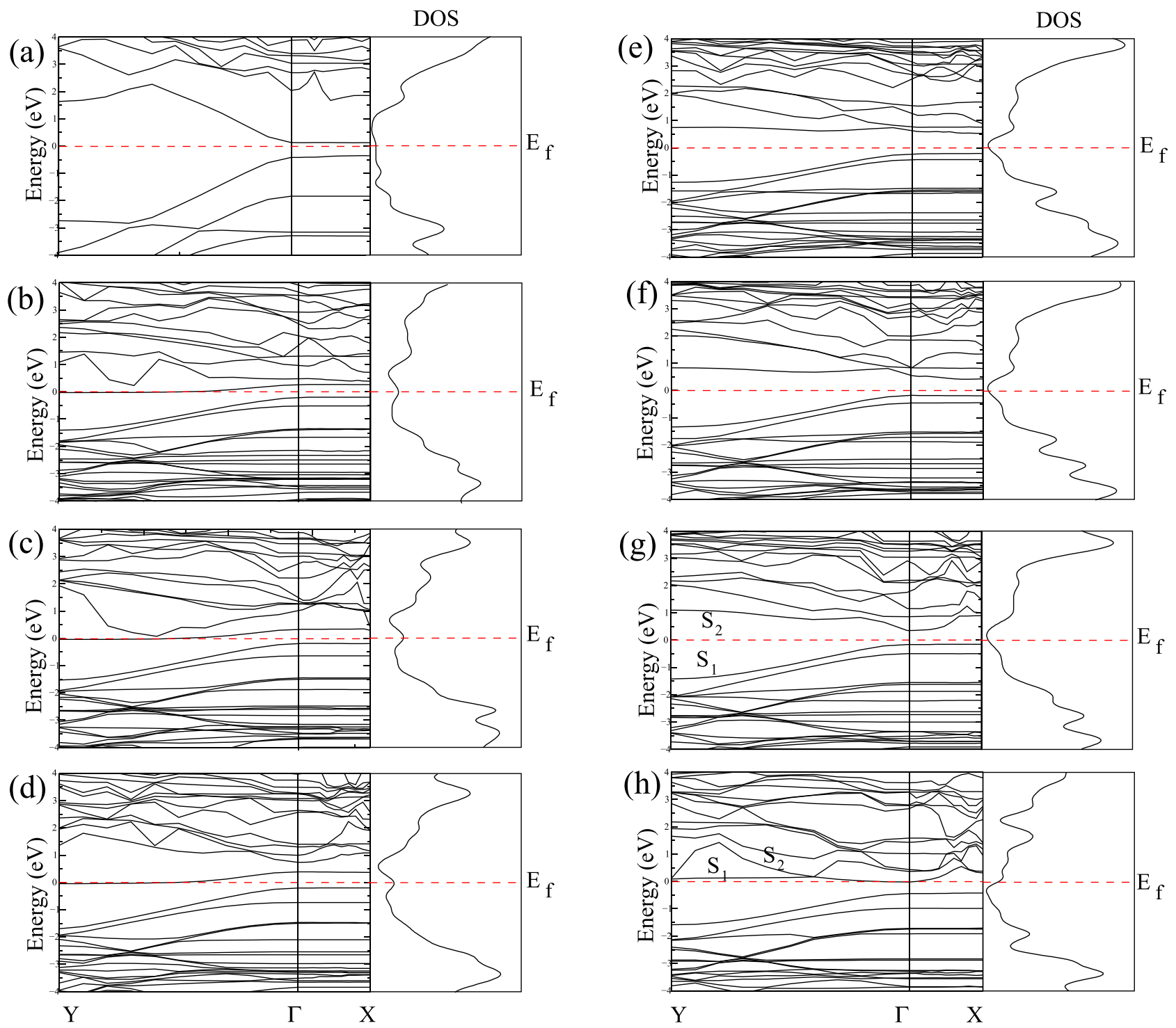

FIG. 3. Density of states around the Fermi level $\left(E_{F}\right)$ for 5-AGNR-C ${ }_{n}(n=4-9)$ with carbon chain of different lengths. For comparison, band structure and den-

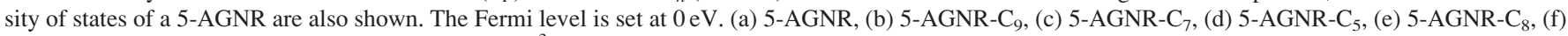
5-AGNR-C 6 , (g) 5-AGNR-C 4 , and (h) 5-AGNR- $\mathrm{C}_{5}-\mathrm{sp}^{3}$.

longest bond length (e.g., bond $b$ ). Both the bond length alteration and the establishment of the largest bond length (single bond) at the ends of a chain are consistent with a recent theoretical study. ${ }^{30}$ The geometrical structures for 4 -AGNR-C 9 and 4-AGNR- $\mathrm{C}_{4}$ are presented in Figs. 1(a) and 1(b). The C-C bond lengths in the chain for the 5-AGNR- $\mathrm{C}_{n}$ are pretty much the same as for 4-AGNR-C ${ }_{n}$. Odd-numbered chains have smaller bond length alteration than even-numbered chains. For even-numbered chains, bonds alternate between $1.30 \AA$ and $1.43 \AA$ for $\mathrm{C}_{8}, 1.29 \AA$ and $1.41 \AA$ for $\mathrm{C}_{6}$, and $1.29 \AA$ and $1.40 \AA$ for $\mathrm{C}_{4}$. For odd-numbered chains, the bond lengths range between $1.37 \AA$ and $1.41 \AA$ for $\mathrm{C}_{9}, 1.38 \AA$ and $1.35 \AA$ for $\mathrm{C}_{7}$, and are explicitly constant at $1.36 \AA$ for $\mathrm{C}_{5}$. The geometrical structures for 5-AGNR- $\mathrm{C}_{5}-s p^{3}$ and 5-AGNR- $\mathrm{C}_{4}$ are presented in Figs. 1(c) and 1(d). The alternating distribution of bond length of short chains is in good agreement with other reported theoretical values. ${ }^{14,16,17,19,31,32}$

The total electronic charge density distribution (Fig. 1) is consistent with the bond length distribution along the chain. The charge density shows an alternating variation, consistent with the distribution of the bond length. The charge density is the minimum along the longest bond $(a)$ and the maximum along the shortest bond $(b)$. The charge density plot in Figs. 1(e)-1(h) indicates that the doublehump feature for bulk diamond has changed to a singlehump feature in linear carbon chains. In accordance with bond lengths and charge densities along the chains, the realistic chemical depiction of bonding configuration is more like $\cdots \mathrm{C}-\mathrm{C} \equiv \mathrm{C}-\mathrm{C} \equiv \mathrm{C} \cdots$ (as in polyyne) for evennumbered chains and $\cdots \mathrm{C}=\mathrm{C}=\mathrm{C}=\mathrm{C} \cdots$ (as in cumulene) for odd-numbered chains.

Electronic band structure calculations were performed along two symmetry directions: $\Gamma-\mathrm{X}$ along the chain and $\Gamma-\mathrm{Y}$ along the AGNR periodicity (i.e., a direction perpendicular to the chain). The calculated electronic band structure, close to the Fermi level and along the $\mathrm{Y}-\Gamma-\mathrm{X}$ direction, and the DOS, for H-terminated 4-AGNRs and 4-AGNR-C ${ }_{n}(n=4-9)$ are presented in Fig. 2. An interesting finding from our calculations is 
TABLE I. The calculated lengths of the C-C bonds labelled $a, b, c, d, e, f, g$, $h, i, j$ (in $\AA$ ) for odd- and even-numbered chains for $4-\mathrm{AGNR}^{-\mathrm{C}_{n}}$ and 5-AGNR-C . $_{n}$

\begin{tabular}{|c|c|c|c|c|c|c|c|c|c|c|}
\hline Model & $a$ & $b$ & $c$ & $d$ & $e$ & $f$ & $g$ & $h$ & $i$ & $j$ \\
\hline 4-AGNR-C ${ }_{9}$ & 1.49 & 1.30 & 1.37 & 1.32 & 1.34 & 1.33 & 1.31 & 1.34 & 1.28 & 1.42 \\
\hline 4-AGNR-C 7 & 1.50 & 1.32 & 1.38 & 1.35 & 1.35 & 1.38 & 1.31 & 1.48 & & \\
\hline 4-AGNR-C ${ }_{5}$ & 1.49 & 1.32 & 1.37 & 1.37 & 1.33 & 1.50 & & & & \\
\hline 4-AGNR-C ${ }_{8}$ & 1.53 & 1.30 & 1.43 & 1.30 & 1.43 & 1.30 & 1.43 & 1.29 & 1.52 & \\
\hline 4-AGNR-C 6 & 1.54 & 1.29 & 1.43 & 1.30 & 1.43 & 1.29 & 1.52 & & & \\
\hline 4-AGNR-C ${ }_{4}$ & 1.48 & 1.28 & 1.40 & 1.28 & 1.49 & & & & & \\
\hline 5-AGNR-C ${ }_{9}$ & 1.59 & 1.33 & 1.45 & 1.37 & 1.41 & 1.41 & 1.37 & 1.44 & 1.33 & 1.57 \\
\hline 5-AGNR-C 7 & 1.50 & 1.32 & 1.38 & 1.35 & 1.35 & 1.37 & 1.31 & 1.47 & & \\
\hline 5-AGNR-C ${ }_{5}$ & 1.48 & 1.32 & 1.36 & 1.36 & 1.32 & 1.48 & & & & \\
\hline 5-AGNR-C ${ }_{8}$ & 1.53 & 1.30 & 1.43 & 1.31 & 1.43 & 1.30 & 1.42 & 1.29 & 1.50 & \\
\hline 5-AGNR-C 6 & 1.51 & 1.29 & 1.42 & 1.29 & 1.41 & 1.29 & 1.50 & & & \\
\hline 5-AGNR-C 4 & 1.48 & 1.29 & 1.40 & 1.28 & 1.48 & & & & & \\
\hline
\end{tabular}

that the electronic structure shows even-odd oscillations with regards to the number of atoms in the chains. Odd-numbered chains are characterized by quite a flat band, which crosses the Fermi energy level in the $\mathrm{Y}-\Gamma$ direction, suggesting a metallic nature (Figs. 2(b)-2(d)). We have found that the lowest unoccupied and highest occupied states are very smooth along the normal to the plane of the CAC and similar level of smoothness is found in the CAC plane. It is found that the band gap of the even-numbered chains of approximately $2.1 \mathrm{eV}$ is smaller than the band gap for 4-AGNR $(2.6 \mathrm{eV})$, but still indicating a clear semiconductor nature.

In order to understand the origin of the states lying closest to the Fermi level (the states labelled $S_{1}$ and $S_{2}$ in Fig. 2), we have plotted their partial charge densities. An examination of Fig. 1 indicates that the $S_{1}$ and $S_{2}$ states are localised on different parts of the chain. For 4-AGNR-C $C_{9}$, we note that $S_{1}$ state is largely derived from the $\pi$ orbitals localized between the $\mathrm{C}-\mathrm{C}$ bonds, except for the central $\mathrm{C}$ atom for which it is a $p_{z}$ orbital. The $\mathrm{S}_{2}$ state is essentially made of $p_{z}$ orbital at alternate $C$ atoms. For 4-AGNR- $C_{4}$, the $S_{1}$ and $S_{2}$ states are made of $\pi / \pi^{*}$ type orbitals formed between neighbouring $\mathrm{C}$ atoms.

Besides the above structures, we have also considered $\mathrm{sp}^{3}$-like bonding geometry between the CAC and the AGNR. According to our results, the $\mathrm{sp}^{3}$ bonding leads to changes in the atomic structure as well as electronic structure of the systems. The 4-AGNR- $\mathrm{C}_{4}$ - $\mathrm{sp}^{3}$ structure does not show any significant difference for the band gap and the dispersion of the band states along the $\mathrm{Y}-\Gamma-\mathrm{X}$ direction. Nevertheless, the band states are shifted above (i.e., to higher energies), verified by the DOS result, so that the electronic structure changes from semiconducting ( $s p^{2}$ structure) to metallic (for the $s p^{3}$ structure). Also, the bonding structure of the chain is changed from polyyne to cumulene. The bonding structure of the chain is not changed for 4-AGNR- $\mathrm{C}_{5}-\mathrm{sp}^{3}$ and its electronic structure is still metallic. The dispersions of various bands have changed and the peak which was located at the Fermi level has moved to right (i.e., to a higher energy).

Figure 3 shows the band structures of 5-AGNR and 5-AGNR- $\mathrm{C}_{n}$ systems. The 5-AGNR structure indicates semiconductor character with a small band gap, in agreement with previous works, ${ }^{2,11}$ and obviously the $s p$ carbon atomic chains cause changes in the electronic structure. Oddnumbered chains have some states in the band gap, which cross the Fermi level along the $\mathrm{Y}-\Gamma$ direction, leading to a peak at the Fermi level. These band states and the peak are indication of the metallic structure. In addition, the band gap is consistent with that for 5-AGNR along the $\Gamma$-X direction. Even-numbered chains show a clear dip in the DOS, but with no state crossing the Fermi level in the $\mathrm{Y}-\Gamma-\mathrm{X}$ direction, which implies a semi-metallic character. From the contour plots for 5-AGNR- $\mathrm{C}_{5}-s p^{3}$, we clearly observe charge accumulation around the bonds labelled $b$ and $e$ for $\mathrm{S}_{1}$ state. For 5-AGNR-C $\mathrm{C}_{4}$, the $p_{z}$ orbitals are located on the bonds labelled $a, c$, and $e$ for the $\mathrm{S}_{1}$ band state and on the bonds labelled $a$, $b, d$, and $e$ for the $\mathrm{S}_{2}$ band state.

We have explored how the structural and electronic properties of such chains change from the $s p^{2}$ to the $s p^{3}$ termination for the 5-AGNR- $\mathrm{C}_{n}$ systems. The bond lengths in the CAC for 5 -AGNR- $\mathrm{C}_{5}-s p^{3}$ are very similar to those for $4-\mathrm{AGNR}-\mathrm{C}_{4}-\mathrm{sp}^{3}$ : for 5 -AGNR- $\mathrm{C}_{5}-s p^{3}$ bond lengths (in $\AA$ ) are ...1.42, 1.37, $1.38,1.42 \ldots$ and for $4-\mathrm{AGNR}_{-} \mathrm{C}_{4}-\mathrm{sp}^{3} \ldots 1.35,1.36,1.36$. Also, the chemical nature of the bond is changed from the polyyne to cumulene for 5-AGNR- $\mathrm{C}_{4}-\mathrm{sp}^{3}$ just like 4-AGNR-C $\mathrm{C}_{4}-\mathrm{sp}^{3}$, but there is no significant change in the electronic structure, which still remains semi-metal. The effect of $\mathrm{sp}^{2}$ versus $\mathrm{sp}^{3}$ bonding of the CAC with the AGNR can be clearly noted from an inspection of the electronic band structure and DOS results presented in panels (d) and (h) in Fig. 3. There are more electronic states near the Fermi level for the $\mathrm{sp}^{3}$ configuration. The DOS features have also changed in the energy range $E_{F} \pm 2 \mathrm{eV}$. The clearly observed peak at $E_{F}$ for the $\mathrm{sp}^{2}$ termination has been changed to a shoulder-like structure with peaks at $-1.94 \mathrm{eV}$ and $1.67 \mathrm{eV}$ for the $\mathrm{sp}^{3}$ termination. We thus can conclude that the ending of sp-carbon chains modifies the electronic structure, specially for odd-numbered chains, in agreement with a previous theoretical analysis. ${ }^{33}$

It is generally thought, on theoretical grounds, that linear CACs should exhibit good ballistic conductance. Due to the metallic character and good conductance, ZGNRs are chosen as the electrodes and the transport properties of chain-ZGNR junctions are extensively studied by several research groups. ${ }^{16-20}$ However, AGNRs are not choosen as the electrodes because of their semiconducting or semimetallic nature, providing poor conductance. Theoretical results for transport along GNR-CAC-GNR have recently been presented. Shen et al. ${ }^{19}$ suggest that for ZGNR-CAC-ZGNR with short CAC, ballistic transport should be observed for odd-numbered chains but not for even-numbered chains.

In the present study, we have investigated the electronic structure of the AGNRs ended rigid short CACs around the Fermi level. The dependence of the DOS at $E_{F}$ on the number of $\mathrm{C}$ atoms in the chain, presented in Figure 4, shows oscillatory behavior with the number of carbon atoms in the chain. As high DOS near $E_{F}$ leads to high conductance, ${ }^{16,19}$ we can conclude that odd numbered chains should have higher conductivity and even-numbered chains should prove poor conductors. For a given $N_{a}$ AGNR-CAC- $N_{a}$ AGNR, the DOS at $E_{F}$ generally increases with the number of atoms in the odd-numbered CAC. Our work thus predicts that the 

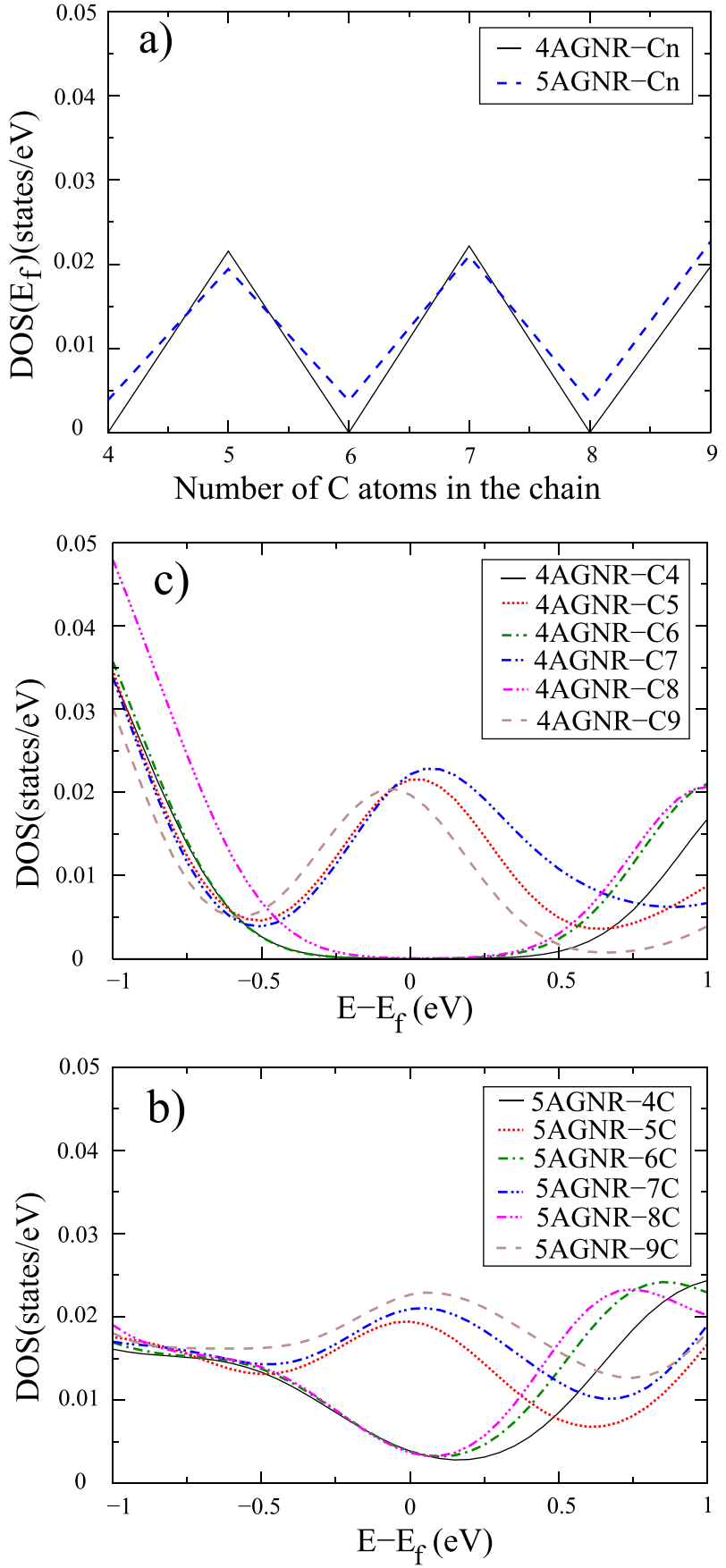

FIG. 4. (a) Density of states at the Fermi level $\left(E_{F}\right)$ as a function of the number of the carbon atoms in the chain for 4-AGNR- $\mathrm{C}_{n}$ and 5-AGNR- $\mathrm{C}_{n}$ $(n=4-9)$. (b) Normalized DOS around the fermi level for 4-AGNR-C $\mathrm{C}_{n}$ and (c) 5-AGNR-C ${ }_{n}(n=4-9)$.

conducting behaviour of short CACs held between AGNRs is similar to that of short CACs held between ZGNRs. Our results also suggest that for short $\mathrm{CACs}$ with odd number of atoms $\left(\mathrm{C}_{n}\right)$, the conductance of $5 \mathrm{AGNRS}-\mathrm{C}_{n}-5 \mathrm{AGNR}$ should be a little lower than that of 4 AGNRS-C ${ }_{n}-4$ AGNR.

\section{SUMMARY}

Inspirated by recent experiments, we have investigated the atomic and electronic properties of short CACs held rigidly between AGNRs using a first-principles theoretical procedure. Our calculations have revealed that oddnumbered chains are metallic in nature, with chemical bonding more like $\cdots \mathrm{C}=\mathrm{C}=\mathrm{C}=\mathrm{C} \cdots$ (as in cumulene). However, even-numbered chains held between 4-AGNR show semiconductor structure and semi-metallic nature when held between 5-AGNR, with chemical bonding more like $\cdots \mathrm{C}-\mathrm{C} \equiv \mathrm{C}-\mathrm{C} \equiv \mathrm{C} \cdots$ (as in polyyne). Our results suggest that the type of attachment between the chain and the ribbon (i.e., $\mathrm{sp}^{2}$ or $\mathrm{sp}^{3}$ ) modifies the electronic structure, specially for odd-numbered chains.

\section{ACKNOWLEDGMENTS}

The calculations reported here were performed using the University of Exeter's SGI Altix ICE 8200 supercomputer.

${ }^{1}$ K. Nakada, M. Fujita, G. Dresselhaus, and M. S. Dresselhaus, Phys. Rev. B 54, 17954 (1996).

${ }^{2}$ Y.-W. Son, M. L. Cohen, and S. G. Louie, Phys. Rev. Lett. 97, 216803 (2006).

${ }^{3}$ M. Y. Han, B. Ozyilmaz, Y. Zhang, and P. Kim, Phys. Rev. Lett. 98, 206805 (2007).

${ }^{4}$ F. Schedin, A. K. Geim, S. V. Morozov, E. W. Hill, P. Blake, M. I. Katsnelson, and K. S. Novoselov, Nature Mater. 6, 652 (2007).

${ }^{5}$ T. B. Martins, R. H. Miwa, A. J. R. Silva, and A. Fazzio, Phys. Rev. Lett. 98, 196803 (2007).

${ }^{6}$ M. Fujita, K. Wakabayashi, K. Nakada, and K. Kusakabe, J. Phys. Soc. Jpn. 65, 1920 (1996).

${ }^{7}$ K. Wakabayashi, M. Fujita, H. Ajiki, and M. Sigrist, Phys. Rev. B 59, 8271 (1999).

${ }^{8}$ M. Ezawa, Phys. Rev. B 73, 045432 (2006).

${ }^{9}$ S. Okada, Phys. Rev. B 77, 041408(R) (2008).

${ }^{10}$ L. Brey and H. A. Fertig, Phys. Rev. B 73, 235411 (2006).

${ }^{11}$ D. Lu, Y. Song, Z. Yang, and G. Li, Appl. Surf. Sci. 257, 6440-6444 (2011).

${ }^{12}$ P. Shemella, Y. Zhang, and M. Mailman, Appl. Phys. Lett. 91, 042101 (2007).

${ }^{13}$ Y. Miyamoto, K. Nakada, and M. Fujita, Phys. Rev. B 59, 9858 (1999).

${ }^{14}$ C. Jin, H. Lan, L. Peng, K. Suenaga, and S. Iijima, Phys. Rev. Lett. 102, 205501 (2009).

${ }^{15}$ A. Chuvilin, J. C. Meyer, G. Algate-Siller, and U. Kaiser, New J. Phys. 11, 083019 (2009).

${ }^{16}$ N. D. Lang and Ph. Avouris, Phys. Rev. Lett. 81, 3515 (1998).

${ }^{17}$ N. D. Lang and Ph. Avouris, Phys. Rev. Lett. 84, 358 (2000).

${ }^{18}$ N. D. Lang and Ph. Avouris, Phys. Rev. B. 62, 7325 (2000).

${ }^{19}$ L. Shen, M. Zeng, S.-W. Yang, C. Zhang, X. Wang, and Y. Feng, J. Am. Chem. Soc. 132, 11481 (2010).

${ }^{20}$ Y. Xu, B.-J. Wang, S.-H. Ke, W. Yang, and A. Z. Alzahrani, J. Chem. Phys. 137, 104107 (2012).

${ }^{21}$ A. Calzolari, N. Marzari, I. Souza, and M. B. Nardelli, Phys. Rev. B 69, 035108 (2004).

${ }^{22}$ D. M. Ceperley and B. J. Alder, Phys. Rev. Lett. 45, 566 (1980).

${ }^{23}$ J. P. Perdew and A. Zunger, Phys. Rev. B 23, 5048 (1981).

${ }^{24}$ X. Gonze, R. Stumpf, and M. Scheffler, Phys. Rev. B 44, 8503 (1991).

${ }^{25}$ L. Kleinman and D. M. Bylander, Phys. Rev. Lett. 48, 1425 (1982).

${ }^{26}$ W. Kohn and L. Sham, Phys. Rev. 140, A1133 (1965).

${ }^{27}$ H. J. Monkhorst and J. D. Pack, Phys. Rev. B 13, 5188 (1976).

${ }^{28}$ H. Hosoya, H. Kumazaki, K. Chida, M. Ohuchi, and Y. D. Gao, Pure Appl. Chem. 62, 445 (1990).

${ }^{29}$ J. Hunter, H. P. Luthi, and F. Diederich, J. Am. Chem. Soc. 116, 750 (1994).

${ }^{30}$ E. Hobi, Jr., R. B. Pontes, A. Fazzio, and A. J. R. Silva, Phys. Rev. B 81, 201406(R) (2010).

${ }^{31}$ K. H. Khoo, J. B. Neaton, Y. W. Son, M. L. Cohen, and S. G. Louie, Nano Lett. 8, 2900 (2008)

${ }^{32}$ Z. Zanolli, G. Onida, and J.-C. Charlier, ACS Nano 4, 5174 (2010).

${ }^{33}$ L. Ravagnan, N. Manini, E. Cinquanta, G. Onida, D. Sangalli, C. Motta1, M. Devetta, A. Bordoni, P. Piseri, and P. Milani, Phys. Rev. Lett. 102, 245502 (2009). 